\title{
Identification of suitable reference genes for gene expression studies in rat skeletal muscle following sciatic nerve crush injury
}

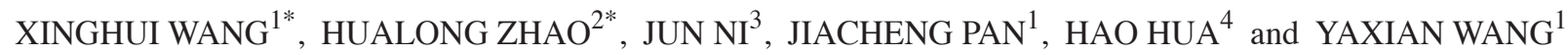 \\ ${ }^{1}$ Key Laboratory of Neuroregeneration of Jiangsu and Ministry of Education, \\ Co-innovation Center of Neuroregeneration, Nantong University, Nantong, Jiangsu 226001; \\ ${ }^{2}$ Jiangsu Provincial Key Laboratory of Coastal Wetland Bioresources and Environmental Protection, \\ Yancheng Teachers' University, Yancheng, Jiangsu 224051; ${ }^{3}$ Department of Rehabilitation, Affiliated Hospital of \\ Nantong University; ${ }^{4}$ Department of Medicine, Xinglin College, Nantong University, Nantong, Jiangsu 226001, P.R. China
}

Received July 6, 2018; Accepted February 28, 2019

DOI: $10.3892 / \mathrm{mmr} .2019 .10102$

\begin{abstract}
Reverse transcription-quantitative polymerase chain reaction (RT-qPCR) is a molecular biological method used to assess gene expression characterized by high simplicity, effectiveness, specificity and sensitivity. The selection of a suitable reference gene for normalization is critical for the accuracy of quantitative results. Peripheral nerve injury is a common clinical disorder that affects multiple tissues and organs, including peripheral nerves, neurons and the innervated muscles. Numerous genes are differentially expressed in skeletal muscles during muscle denervation and reinnervation following peripheral nerve injury. The identification of a suitable reference gene in innervated muscles following nerve injury may improve the understanding of the alterations in gene expression in the processes of peripheral nerve repair and regeneration. Therefore, in the present study, by using a rat sciatic nerve crush model, the expression levels of various housekeeping genes were examined. In particular, the expression levels of 13 housekeeping genes, including $18 \mathrm{~S}$ ribosomal RNA, actin $\beta$, ankyrin repeat domain 27, cyclophilin A, GAPDH, hypoxanthine phosphoribosyltransferase 1 (HPRT1), mitochondrial ribosomal protein L10, phosphoglycerate kinase 1, RPTOR independent companion of mammalian target of rapamycin complex 2, TATA-box binding protein, ubiquitin $\mathrm{C}$, UBX domain protein 11 and tyrosine 3-monooxygenase/tryptophan 5-monooxygenase
\end{abstract}

Correspondence to: Dr Yaxian Wang, Key Laboratory of Neuroregeneration of Jiangsu and Ministry of Education, Co-innovation Center of Neuroregeneration, Nantong University, Building No. 7, 19 Qixiu Road, Nantong, Jiangsu 226001, P.R. China E-mail:wyx1984@ntu.edu.cn

*Contributed equally

Key words: rat sciatic nerve injury, gastrocnemius muscle, reference gene, geNorm, NormFinder, normalization activation protein $\zeta$, were investigated in gastrocnemius muscles. The geNorm and NormFinder analyses suggested that the expression level of HPRT1 was particularly stable in gastrocnemius muscles following rat sciatic nerve crush injury. Therefore, HPRT1 may be used as a reference gene for the normalization of gene expression data generated by RT-qPCR.

\section{Introduction}

Reverse transcription-quantitative polymerase chain reaction (RT-qPCR) is a molecular biological method used to measure the levels of gene expression. RT-qPCR is characterized by high simplicity, effectiveness, specificity and sensitivity $(1,2)$. However, the absolute measures derived from RT-qPCR analyses may be affected by multiple factors, including the initial loading amount of RNA, the RNA quality and integrity, the efficiency of the enzymes used in RT-qPCR and the discrepancy in the expression levels of various RNAs between tissues and cells (3). To obtain reliable measurements, it is important to use an internal control gene for normalization. Housekeeping genes are genes involved in fundamental biological processes and are frequently expressed at constant levels in various cell types $(4,5)$. Therefore, housekeeping genes are frequently used as internal controls for the normalization of gene expression. However, accumulating evidence has demonstrated that the endogenous expression levels of various housekeeping genes may be variable under certain physiological and pathological conditions and, to the best of our knowledge, no housekeeping gene exhibits a stable expression level under every condition (6-8).

Peripheral nerve injury is a clinical disorder that affects 13-23 patients per 100,000 per year in developed countries (9). Following nerve injury, peripheral nerves undergo multiple regenerative processes including Wallerian degeneration of the distal nerve stump, the regrowth and regeneration of axons, and reinnervation (10). Our previous high-throughput study identified that the expression levels of multiple housekeeping genes were differentially expressed in injured sciatic nerve samples and dorsal root ganglion (DRG) samples following rat 
sciatic nerve injury, and suitable reference genes for RT-qPCR normalization were selected (11-13).

In addition to peripheral nerves and neurons, target skeletal muscles may exhibit denervation and reinnervation following peripheral nerve injury. The denervation and reinnervation processes are involved in the preservation of the biological functions of innervated muscles $(14,15)$. Gene expression analyses are frequently used to investigate the denervation and reinnervation processes of target muscles. Therefore, it is important to identify suitable reference genes in innervated muscles following nerve injury.

Therefore, in the present study, a rat sciatic nerve crush model was employed. Subsequently, gastrocnemius muscles were collected and the cycle quantification $(\mathrm{Cq})$ values of $18 \mathrm{~S}$ ribosomal RNA (18S), actin $\beta$ (ACTB), ankyrin repeat domain 27 (ANKRD27), cyclophilin A (CYPA), GAPDH, hypoxanthine phosphoribosyltransferase 1 (HPRT1), mitochondrial ribosomal protein L10 (MRPL10), phosphoglycerate kinase 1 (PGK1), RPTOR independent companion of mammalian target of rapamycin complex 2 (RICTOR), TATA-box binding protein (TBP), ubiquitin C (UBC), UBX domain protein 11 (UBXN11) and tyrosine 3-monooxygenase/tryptophan 5-monooxygenase activation protein $\zeta$ (YWHAZ) were measured. The expression level of HPRT1 exhibited the highest stability among the housekeeping genes analyzed in the present study, whereas PGK1 exhibited the highest variability.

\section{Materials and methods}

Animal surgery. A total of 30 female Sprague-Dawley rats (age, 2 months; weight, 180-220 g) were purchased from The Experimental Animal Center of Nantong University (Nantong, China) and subjected to sciatic nerve crush injury as previously described (11). Rats were anaesthetized by injecting $2 \mathrm{ml} / \mathrm{kg}$ Equithesin (Sigma-Aldrich; Merck KGaA, Darmstadt, Germany), as previously described (12), corresponding to $17 \mathrm{mg} / \mathrm{kg}$ sodium pentobarbital, $42 \mathrm{mg} / \mathrm{kg}$ magnesium sulfate and $85 \mathrm{mg} / \mathrm{kg}$ trichloroacetaldehyde monohydrate) $(11,16)$. Anesthesia was assessed by loss of pedal reflexes. Rat left sciatic nerves were crushed with forceps for $10 \mathrm{sec}$, three times. To minimize the discomfort and possible painful mechanical stimulation, the rats were housed in large cages with sawdust bedding post-surgery and maintained in a controlled environment under constant temperature of $25^{\circ} \mathrm{C}$ and relative humidity of $40-70 \%$, with a $12 \mathrm{~h}$ light/dark cycle and free access to food and water. Rats were sacrificed 24 h, 4 days, or 1, 2, 3, 4 or 8 weeks following surgery. Gastrocnemius muscles from the left (injured) side and the right (uninjured) side were collected. Control rats were anesthetized without sciatic nerve crushing (sham surgery). All animal procedures were ethically approved by The Administration Committee of Experimental Animals of The Jiangsu Province (Jiangsu, China).

Compound muscle action potential (CMAP) recording. CMAP recording was performed at 1, 2, 4 and 8 weeks following surgery. Following anesthesia, the left sciatic nerves of the rats were analyzed. The active and reference electrodes were placed into the medial belly and Achilles tendon of gastrocnemius, respectively. The stimulating electrode was placed sequentially in the proximal and distal sciatic nerves. A 2-mA electric stimulus was applied to the stimulating electrode to stimulate CMAP responses. CMAP readings at the proximal and distal sides were recorded using a portable digital MYTO electromyographic machine (Esaote SpA, Genoa, Italy) with Galileo NT System Software (EB Neuro SpA, Florence, Italy).

Muscle weight measurement. Gastrocnemius muscles on the left (injured) side and the right (uninjured) side were collected and weighed in healthy control rats and nerve-injured rats at $24 \mathrm{~h}, 4$ days, or 1, 2, 3, 4 or 8 weeks following nerve crush injury. The wet weight of the muscle on the left side was divided by the wet weight of the muscle on the right side to calculate the ratio of injured to uninjured muscle wet weight.

Immunohistochemistry staining and Masson's trichrome staining. Left-side gastrocnemius muscles were collected and fixed in $4 \%$ paraformaldehyde for $12 \mathrm{~h}$ at $4^{\circ} \mathrm{C}$, embedded in Tissue-Tek ${ }^{\circledR}$ optimal cutting temperature compound (Sakura Finetek USA, Inc., Torrance, CA, USA), and cut into $25-\mu \mathrm{m}$ thick longitudinal sections using a cryostat microtome at $-15^{\circ} \mathrm{C}$ (Leica Microsystems GmbH, Wetzlar, Germany). Sections were washed with PBS, blocked with normal goat serum (Invitrogen; Thermo Fisher Scientific, Inc., Waltham, MA, USA) for $1 \mathrm{~h}$ at $37^{\circ} \mathrm{C}$ and incubated with anti-neurofilament $\mathrm{H}$ (NEFH) antibody (1:200; cat.no. SAB4200747; Sigma-Aldrich; Merck $\mathrm{KGaA}$ ) overnight at $4^{\circ} \mathrm{C}$. Tissue sections were washed, incubated with secondary antibodies conjugated with Alexa Fluor 488 (1:400; cat. no. ab150113; Abcam, Cambridge, MA, USA) and tetramethylrhodamine-labeled $\alpha$-Bungarotoxin (1:300; cat. no. 203980; Sigma-Aldrich; Merck KGaA) for $2 \mathrm{~h}$ at room temperature and observed under a fluorescence microscope at x100 magnification (Axio Imager M2; Carl Zeiss AG, Oberkochen, Germany).

In addition, after fixation in $4 \%$ paraformaldehyde for $12 \mathrm{~h}$ at $4^{\circ} \mathrm{C}$, gastrocnemius muscles were embedded in paraffin and cut into transverse sections (6- $\mu \mathrm{m}$ thick). Tissue sections were subjected to Masson's trichrome staining at room temperature for about $1 \mathrm{~h}$, to stain muscle fibers in red, collagen fibers in blue and cell nuclei in black, and observed using light microscopy at x200 magnification (Axio Imager M2; Carl Zeiss AG, Oberkochen, Germany). Fiber cross-sectional area (CSA) was calculated using Leica QWin version 3 Image Analysis System Software (Leica Microsystems $\mathrm{GmbH}$ ).

$R T-q P C R$. Total RNA was isolated from left-side gastrocnemius muscles using TRIzol ${ }^{\circledR}$ Reagent (Thermo Fisher Scientific, Inc.), purified with RNeasy spin columns (Qiagen, Inc., Valencia, CA, USA) to remove DNA and reverse transcribed to cDNA with the PrimeScript reagent kit, $37^{\circ} \mathrm{C}$ for $15 \mathrm{~min}$ and $85^{\circ} \mathrm{C}$ for $5 \mathrm{sec}$, according to the manufacturer's protocol (Takara Biotechnology Co., Ltd., Dalian, China). RT-qPCR was performed using the QuantiNova SYBR Green PCR kit (Qiagen, Inc.) and the StepOne ${ }^{\mathrm{TM}}$ real-time PCR System (Applied Biosystems; Thermo Fisher Scientific, Inc.). An initial denaturation of $10 \mathrm{~min}$ at $95^{\circ} \mathrm{C}$ was followed by 40 cycles of $95^{\circ} \mathrm{C}$ for $15 \mathrm{sec}$ and $60^{\circ} \mathrm{C}$ for $30 \mathrm{sec}$. The $\mathrm{Cq}$ values 


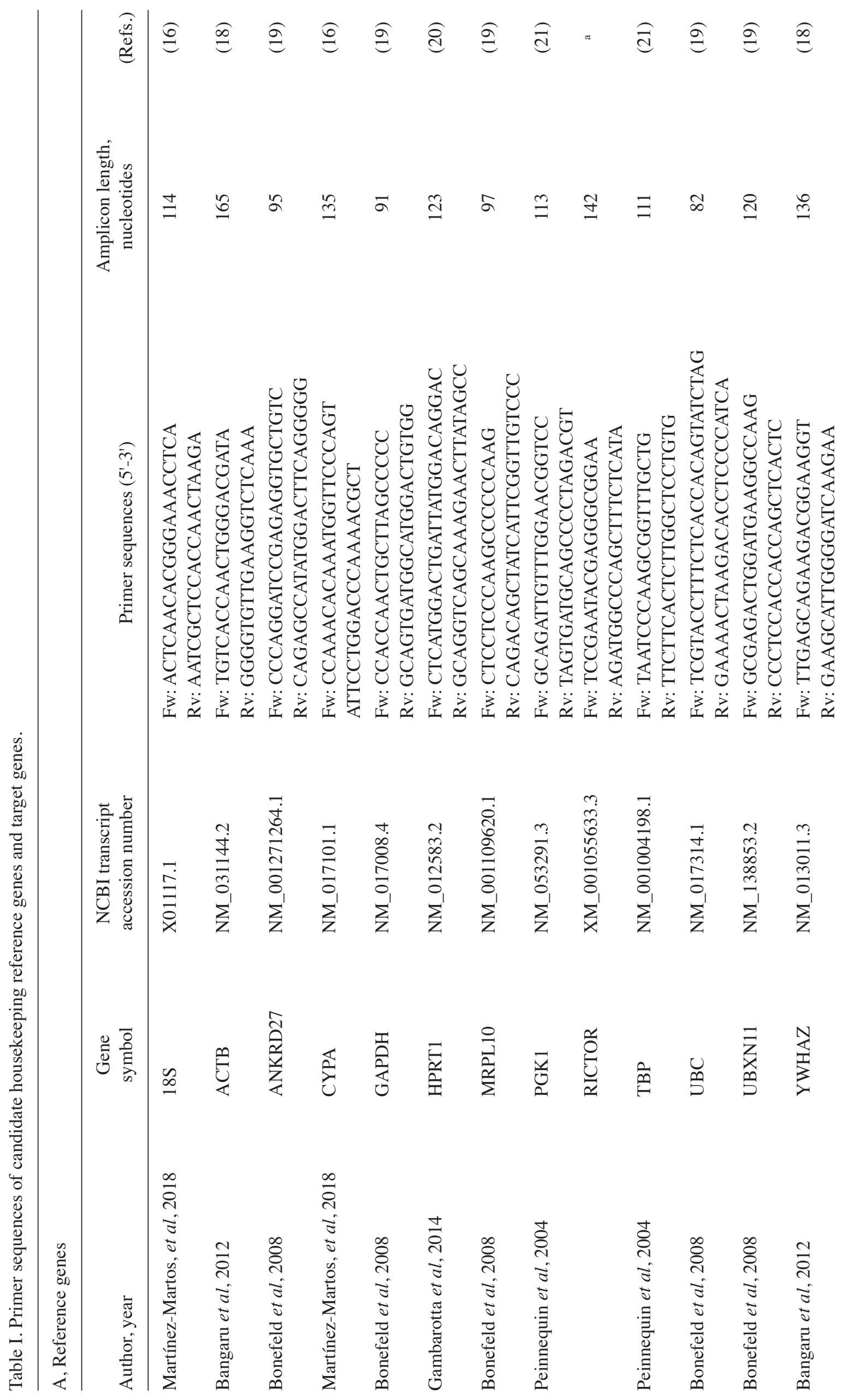


for the candidate reference genes 18S, ACTB, ANKRD27, CYPA, GAPDH, HPRT1, MRPL10, PGK1, RICTOR, TBP, UBC, UBXN11 and YWHAZ were determined for subsequent geNorm and NormFinder analyses. The $\mathrm{Cq}$ values for the target genes F-box protein 32 (FBXO32) and tripartite motif containing 63 (TRIM63) were additionally assessed and the expression levels of FBXO32 and TRIM63 were calculated using the $2^{-\Delta \Delta C q}$ method (17). The quality of the RT-qPCR analysis was confirmed by a single melt-curve peak, representing a single amplification product. Primer sequences (listed in Table I) were obtained from previous publications (18-23) or designed using Primer Express ${ }^{\circledR}$ software (v3.0.1; Thermo Fisher Scientific, Inc.).

PCR amplification efficiency analysis. The amplification efficiency analysis was performed by producing serial dilutions of the DNA sample. Subsequently, the Cq values were plotted on a logarithmic scale along with corresponding concentrations. Using a linear regression curve through, the slope of the trend line was calculated. Finally, efficiency was defined using the equation: $\mathrm{E}=-1+10^{(-1 / \mathrm{slope})}$. Typically, desired amplification efficiencies range from 90 to $110 \%$ (https://biosistemika. com/blog/qpcr-efficiency-over-100/).

geNorm and NormFinder analysis. Cq values for candidate reference genes were subjected to geNorm analysis (v3.4; https://genorm.cmgg.be/), a Visual Basic for plug-in for Microsoft Excel 2007 (Microsoft Corporation, Redmond, WA, USA), that was used to calculate the stability of the expression levels of the reference genes. Gene expression stability (M value) was determined on the basis of non-normalized expression levels of internal reference genes according to a previous study (6). The normalization factor (pairwise variation value, $\mathrm{Vn} / \mathrm{n}+1$ ) was calculated based on the geometric mean of multiple reference genes (6). The stability values of candidate reference genes were also measured using the NormFinder analysis (v19, https://moma. $\mathrm{dk} /$ normfinder-software), a model-based variance estimation approach of Microsoft Excel (Microsoft Corporation), according to a previous study (3).

Statistical analysis. Data are presented as the mean \pm standard deviation. Each independent experiment was repeated three times. Statistical analysis was performed using GraphPad Prism 6.0 (GraphPad Software, Inc., La Jolla, CA, USA). The linear association between two variables was assessed by calculating the correlation coefficient $\mathrm{R}^{2}$, ranging from 0 to 1 . Differences between rats in the injury and normal control groups were tested using one-way analysis of variance followed by Dunnett's multiple comparisons test. $\mathrm{P}<0.05$ was considered to indicate a statistically significant difference.

\section{Results}

Gastrocnemius muscles exhibit denervation and reinnervation following sciatic nerve crush injury. To investigate muscle denervation and reinnervation, morphological and physiological parameters of gastrocnemius muscles were examined in normal control rats and in rats subjected to sciatic nerve 
A

(Con)

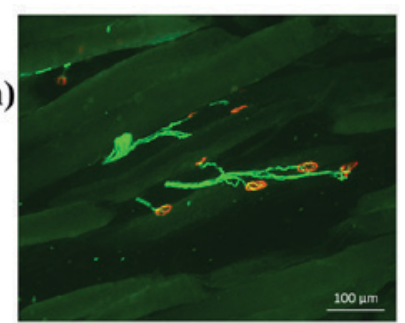

D

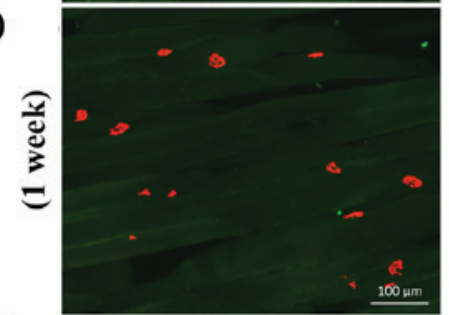

G

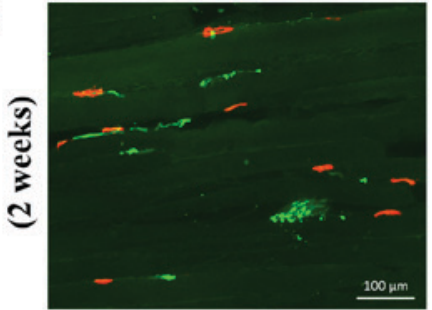

$J$

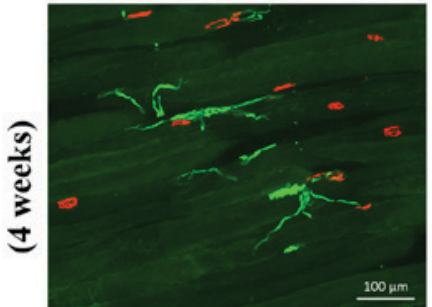

M
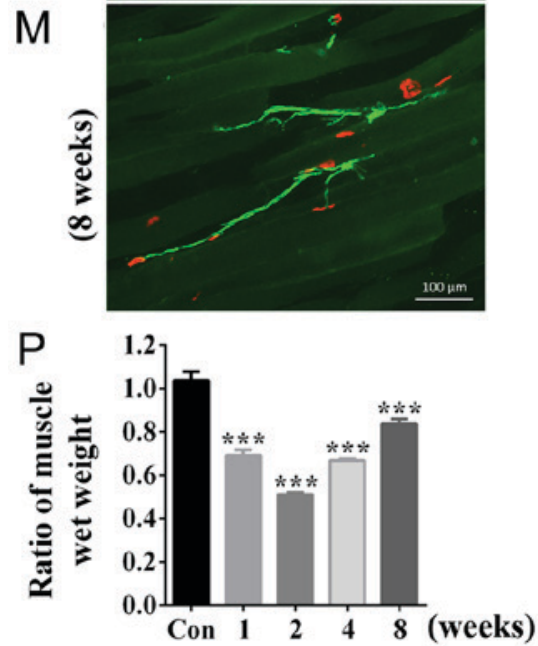

B

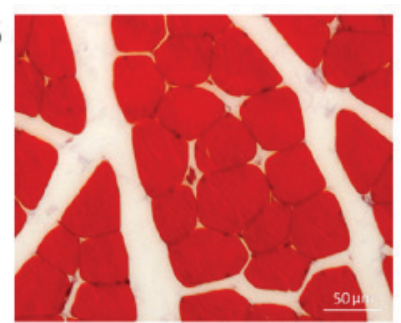

$\mathrm{E}$

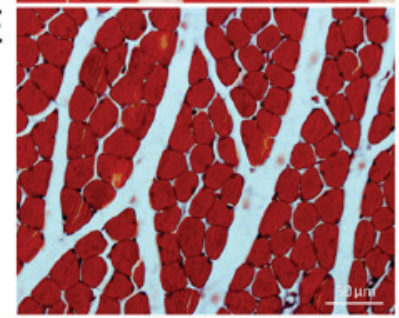

Hinter
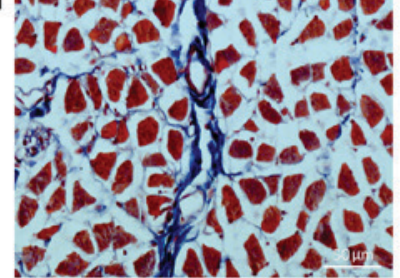

K
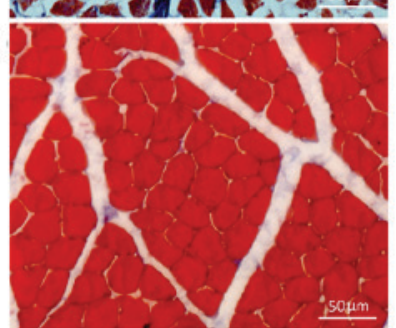

$\mathrm{N}$

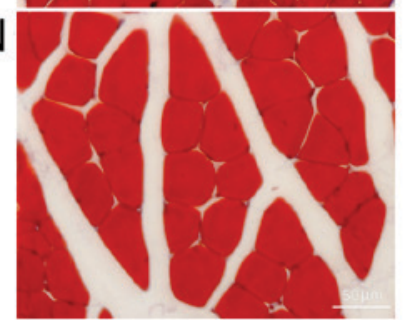

Q ${ }_{1600}^{1600}$

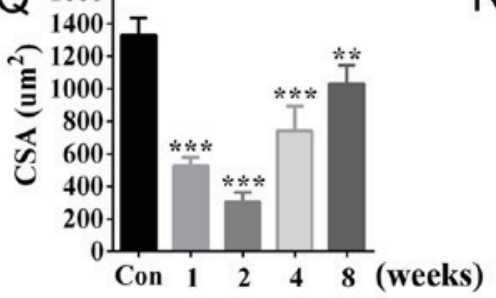

C

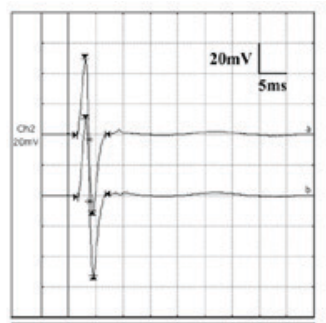

F

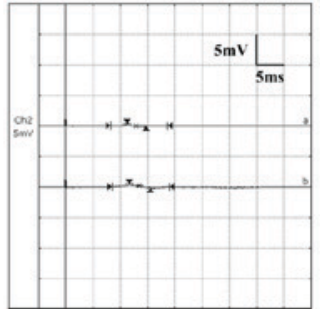

I

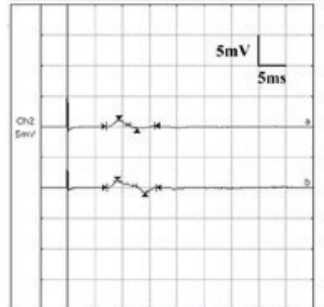

L
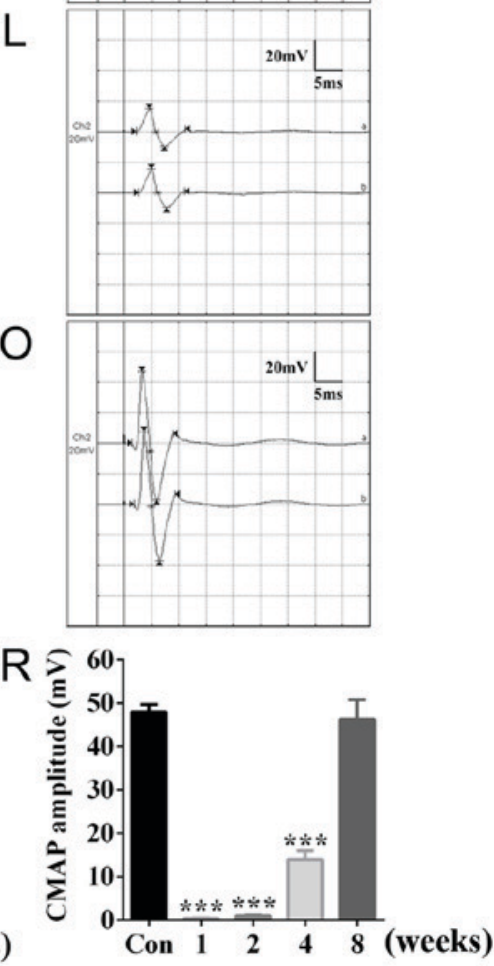

Figure 1. Morphological and electrophysiological alterations in rat gastrocnemius muscles following sciatic nerve crush injury. Gastrocnemius muscles of control rats were analyzed using (A) immunohistochemistry, (B) Masson's trichrome staining and (C) CMAP recording. Gastrocnemius muscles at 1 week following sciatic nerve injury were analyzed using (D) immunohistochemistry, (E) Masson's trichrome staining and (F) CMAP recording. Gastrocnemius muscles at 2 weeks following sciatic nerve injury were analyzed using (G) immunohistochemistry, (H) Masson's trichrome staining and (I) CMAP recording. Gastrocnemius muscles at 4 week following sciatic nerve injury were analyzed using (J) immunohistochemistry, (K) Masson's trichrome staining and (L) CMAP recording. Gastrocnemius muscles at 8 weeks following sciatic nerve injury were analyzed using (M) immunohistochemistry, (N) Masson's trichrome staining and (O) CMAP recording. Immunohistochemistry was performed using anti-neurofilament $\mathrm{H}$ (green) and $\alpha$-Bungarotoxin (red); scale bars, $100 \mu \mathrm{m}$. Tissue sections were subjected to Masson's trichrome staining to stain muscle fibers in red, collagen fibers in blue and cell nuclei in black; scale bar, $50 \mu \mathrm{m}$. (P) Ratio of injured to uninjured muscle wet weight ( $\mathrm{n}=4$ in each group) measured in various groups. (Q) CSA ( $\mathrm{n}=4$ in each group) in various groups. (R) CMAP amplitude ( $\mathrm{n}=4$ in each group) in various groups. ${ }^{* *} \mathrm{P}<0.01$ and ${ }^{* * * *} \mathrm{P}<0.001$ vs. corresponding control. CMAP, compound muscle action potential; CSA, cross-sectional area; Con, control.

injury. Staining of the axon marker NEFH and the motor end-plate marker $\alpha$-Bungarotoxin (Fig. 1A) were detected, and the structure of muscle fibers (Fig. 1B) appeared to be intact. Furthermore, normal CMAP amplitude (Fig. 1C) was 
Table II. Amplification efficiencies of candidate housekeeping genes and target genes.

\section{A, Reference genes}

\begin{tabular}{lccc}
\hline Gene symbol & Slope & $\mathrm{R}^{2}$ & Efficiency $(\%)$ \\
\hline 18S & -3.523 & 0.9994 & 92 \\
ACTB & -3.277 & 0.9921 & 102 \\
ANKRD27 & -3.567 & 0.9976 & 91 \\
CYPA & -3.460 & 0.9974 & 95 \\
GAPDH & -3.489 & 0.9998 & 93 \\
HPRT1 & -3.505 & 0.9996 & 93 \\
MRPL10 & -3.518 & 0.9920 & 92 \\
PGK1 & -3.490 & 0.9983 & 93 \\
RICTOR & -3.507 & 0.9963 & 93 \\
TBP & -3.558 & 0.9921 & 91 \\
UBC & -3.433 & 0.9989 & 96 \\
UBXN11 & -3.373 & 0.9994 & 98 \\
YWHAZ & -3.307 & 0.9975 & 101 \\
\hline
\end{tabular}

\section{$\mathrm{B}$, Target genes}

\begin{tabular}{lccc}
\hline Gene symbol & Slope & $\mathrm{R}^{2}$ & Efficiency (\%) \\
\hline FBXO32 & -3.463 & 0.9977 & 94 \\
TRIM63 & -3.450 & 0.9999 & 95 \\
\hline
\end{tabular}

18S, 18S ribosomal RNA; ANKRD27, ankyrin repeat domain 27; CYPA, cyclophilin A; HPRT1, hypoxanthine phosphoribosyltransferase 1; MRPL10, mitochondrial ribosomal protein L10; PGK1, phosphoglycerate kinase 1; RICTOR, RPTOR independent companion of mammalian target of rapamycin complex 2; TBP, TATA-box binding protein; UBC, ubiquitin C; UBXN11, UBX domain protein 11; YWHAZ, tyrosine 3-monooxygenase/tryptophan 5-monooxygenase activation protein $\zeta$; FBXO32, F-box protein 32 ; TRIM63, tripartite motif containing 63; ACTB, actin $\beta$.

observed in the gastrocnemius muscles of uninjured rats. At 1 week following sciatic nerve injury, degenerated axon fragments were not present, and axons were not detectable between muscle fibers (Fig. 1D), muscle fibers were atrophied (Fig. 1E) and CMAP was not be detected (Fig. 1F). At 2 weeks following sciatic nerve injury, a small number of regenerated axons were observed, and a subset of axons initiated reinnervation (Fig. 1G). Masson's trichrome staining results suggested the presence of muscle atrophy and a large number of hyperplastic collagen fibers were observed (Fig. 1H). Low CMAP amplitude was recorded (Fig. 1I) at 2 weeks following injury. Multiple regenerated axons and reinnervated fibers were observed at 4 weeks following nerve injury (Fig. 1J). Additionally, muscle atrophy (Fig. 1K) and CMAP (Fig. 1L) were partially recovered. At 8 weeks following sciatic nerve injury, the number of regenerated axons and reinnervated fibers increased (Fig. 1M), the morphology of muscles (Fig. 1N) and the CMAP profile (Fig. 1O) were similar to the control rats (Fig. 1A-C).

Additionally, the ratio of injured to uninjured muscle wet weight decreased significantly following nerve injury at week 1 and 2, and improved at 4 and 8 weeks (Fig. 1P). CSA exhibited a similar trend (Fig. 1Q), suggesting that the gastrocnemius muscles underwent muscle atrophy following nerve injury. Quantification of the CMAP results suggested that the decreased CMAP amplitude detected at 2 weeks was increased at 4 weeks following nerve injury and was restored to normal levels at 8 weeks following injury (Fig. 1R). Collectively, the present data suggested that the physiological functions of muscles were gradually restored during the process of reinnervation of target muscles.

$\mathrm{Cq}$ values of reference genes in gastrocnemius muscles following sciatic nerve crush injury. Prior to the determination of the $\mathrm{Cq}$ values of the reference genes, the efficiencies of the primers selected for RT-qPCR were examined. Following a serial dilution, the amplifications of the 13 candidate housekeeping genes exhibited linear standard curves with slope values of $\sim-3.322$ and a linear correlation coefficient $\left(R^{2}\right)$ of $\sim 1$ (Table II). The amplification efficiencies of the primer pairs of all housekeeping genes were calculated based on the slope of the linear standard curves. The amplification efficiencies of all primer pairs were between 90 and 102\% (Table II).

Subsequently, RT-qPCR experiments were performed to measure the raw $\mathrm{Cq}$ values of the reference genes. $18 \mathrm{~S}$ exhibited the lowest $\mathrm{Cq}$ value, indicating that the abundance of $18 \mathrm{~S}$ ribosomal RNA gene was the highest in rat gastrocnemius muscles. The $\mathrm{Cq}$ values of GAPDH, a gene encoding an enzyme involved in glycolysis, were decreased compared with other genes. The Cq values of UBXN11 were increased compared with other housekeeping genes, suggesting that its endogenous expression level was the lowest. The $\mathrm{Cq}$ values of the other housekeeping genes ranged between 20 and 30 (Fig. 2). In addition, the $\mathrm{Cq}$ values of the housekeeping genes in gastrocnemius muscles were assessed at various time points following sciatic nerve injury. The Cq values of ANKRD27, HPRT1, MRPL10, RICTOR and TBP exhibited a stable level in normal control rats and nerve-injured rats at various time points. By contrast, the $\mathrm{Cq}$ values of $18 \mathrm{~S}$, ACTB, CYPA, GAPDH, PGK1, UBC, UBXN11 and YWHAZ exhibited variability among time points (Fig. 2).

Reference gene HPRT1 exhibits high stability. The stabilities of the gene expression levels of the candidate reference genes were examined using geNorm analysis. Average expression stability was calculated and $M$ values were assigned to each reference gene. The $\mathrm{M}$ value calculated for each gene was inversely proportional to the stability of the expression level of the corresponding gene. Our previous study identified that all reference genes examined exhibited $M<1.5$, corresponding to the default limit (6). The reference genes ANKRD27 and HPRT1 exhibited decreased M values compared with the other reference genes, suggesting that the expression levels of these two reference genes were the most stable (Fig. 3A). PGK1 presented the highest $\mathrm{M}$ value, corresponding to the lowest stability (Fig. 3A). The normalization factor (pairwise variation value, $\mathrm{V}_{\mathrm{n} / \mathrm{n}+1}$ ) was calculated to assess the optimal numbers of reference genes to use for gene expression normalization. The pairwise variation value $\mathrm{V}_{2 / 3}$ calculated was 0.103 , below the default limit of $\mathrm{V}_{\mathrm{n} / \mathrm{n}+1}=0.15$ (24), suggesting that the two 

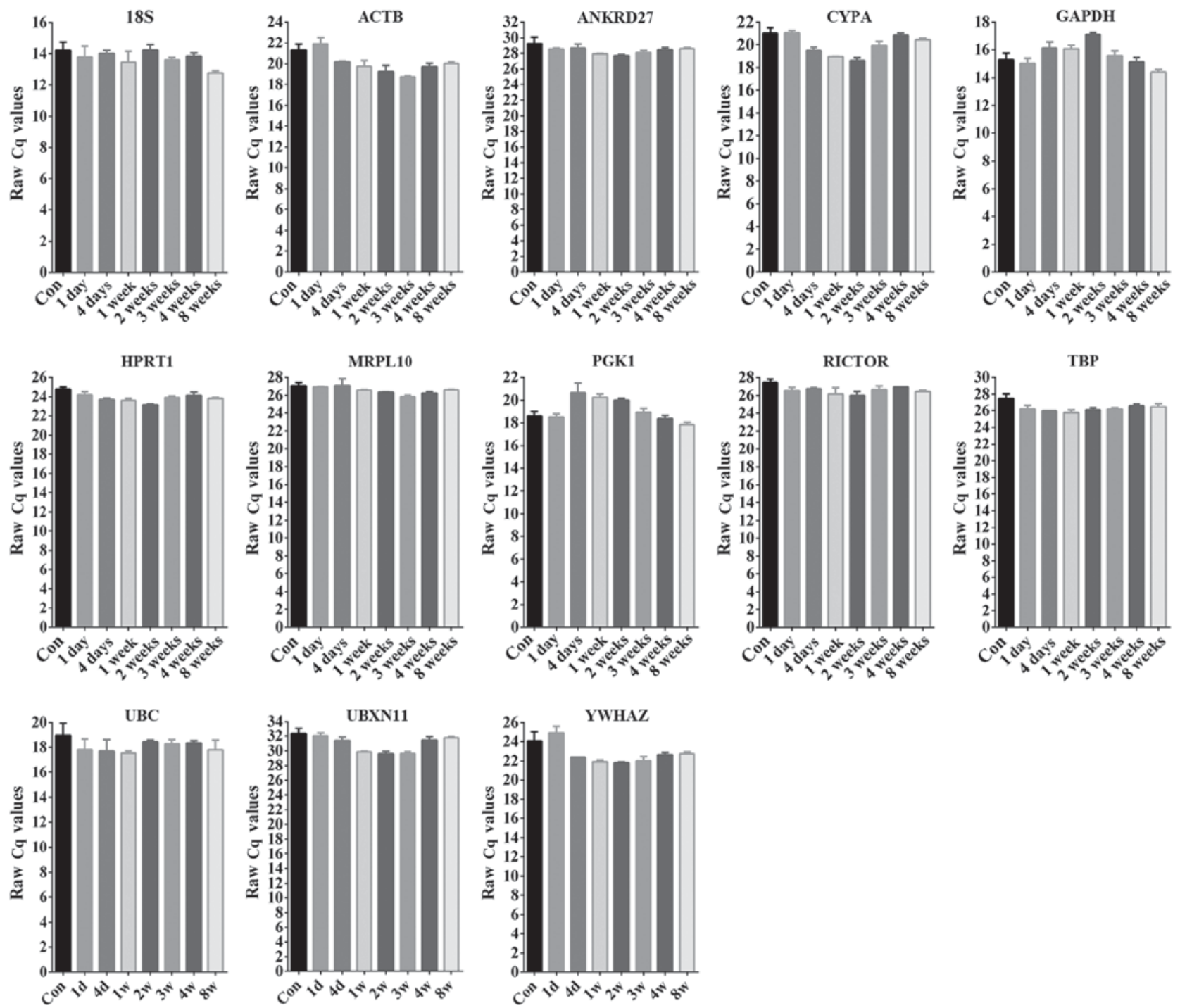

Figure 2. Raw Cq values of candidate reference genes in gastrocnemius muscles. The Cq values of 18S, ACTB, ANKRD27, CYPA, GAPDH, HPRT1, MRPL10, PGK1, RICTOR, TBP, UBC, UBXN11 and YWHAZ in normal control rats and rats subjected to sciatic nerve injury 1, 2, 4 or 8 weeks following injury were assessed by reverse transcription-quantitative polymerase chain reaction ( $\mathrm{n}=3$ in each group). 18S, 18S ribosomal RNA; ANKRD27, ankyrin repeat domain 27; CYPA, cyclophilin A; HPRT1, hypoxanthine phosphoribosyltransferase 1; MRPL10, mitochondrial ribosomal protein L10; PGK1, phosphoglycerate kinase 1; RICTOR, RPTOR independent companion of mammalian target of rapamycin complex 2; TBP, TATA-box binding protein; UBC, ubiquitin C; UBXN11, UBX domain protein 11; YWHAZ, tyrosine 3-monooxygenase/tryptophan 5-monooxygenase activation protein $\zeta$; ACTB, actin $\beta$.

reference genes ANKRD27 and HPRT1 were sufficient to normalize gene expression levels in rat gastrocnemius muscles (Fig. 3B).

The stabilities of the expression levels of the reference genes were further analyzed and ranked using NormFinder. Low stability values were associated with stable expression levels. NormFinder analysis suggested that HPRT1 was the reference gene with the most stable expression level, followed by ANKRD27. The expression level of PGK1 exhibited the lowest stability among the genes examined (Fig. 4). The NormFinder results were consistent with the geNorm results. Collectively, the present results suggested that, in rat gastrocnemius muscles following sciatic nerve injury, the expression levels of HPRT1 and PGK1 exhibited the highest and the lowest stability, respectively.

Determination of gene expression levels is influenced by normalization genes. The influence of distinct reference genes was examined by determining the expression levels of FBXO32 and TRIM63, two genes encoding skeletal muscle atrophy markers $(23,25,26)$. The primer pairs of FBXO32 and TRIM63 exhibited linear standard curves with high efficiency values (94 and 95\%, respectively; Table II). HPRT1 and PGK1 exhibited the lowest and the highest stability, respectively. Therefore, these two genes were used as internal controls to perform RT-qPCR experiments (Fig. 5).

Using HPRT1 as the internal control, the expression levels of FBXO32 were observed to be significantly upregulated in rat gastrocnemius muscles following sciatic nerve injury, exhibiting a maximum at 1 day following injury (Fig. 5A). Similarly, using PGK1 as internal control, the expression levels of FBXO32 were observed to be upregulated following nerve injury. However, the highest $\mathrm{Cq}$ value of FBXO32 was detected at 1 week following injury. Notably, the fold changes of FBXO32 were identified to vary depending on the reference gene selected (Fig. 5B). 

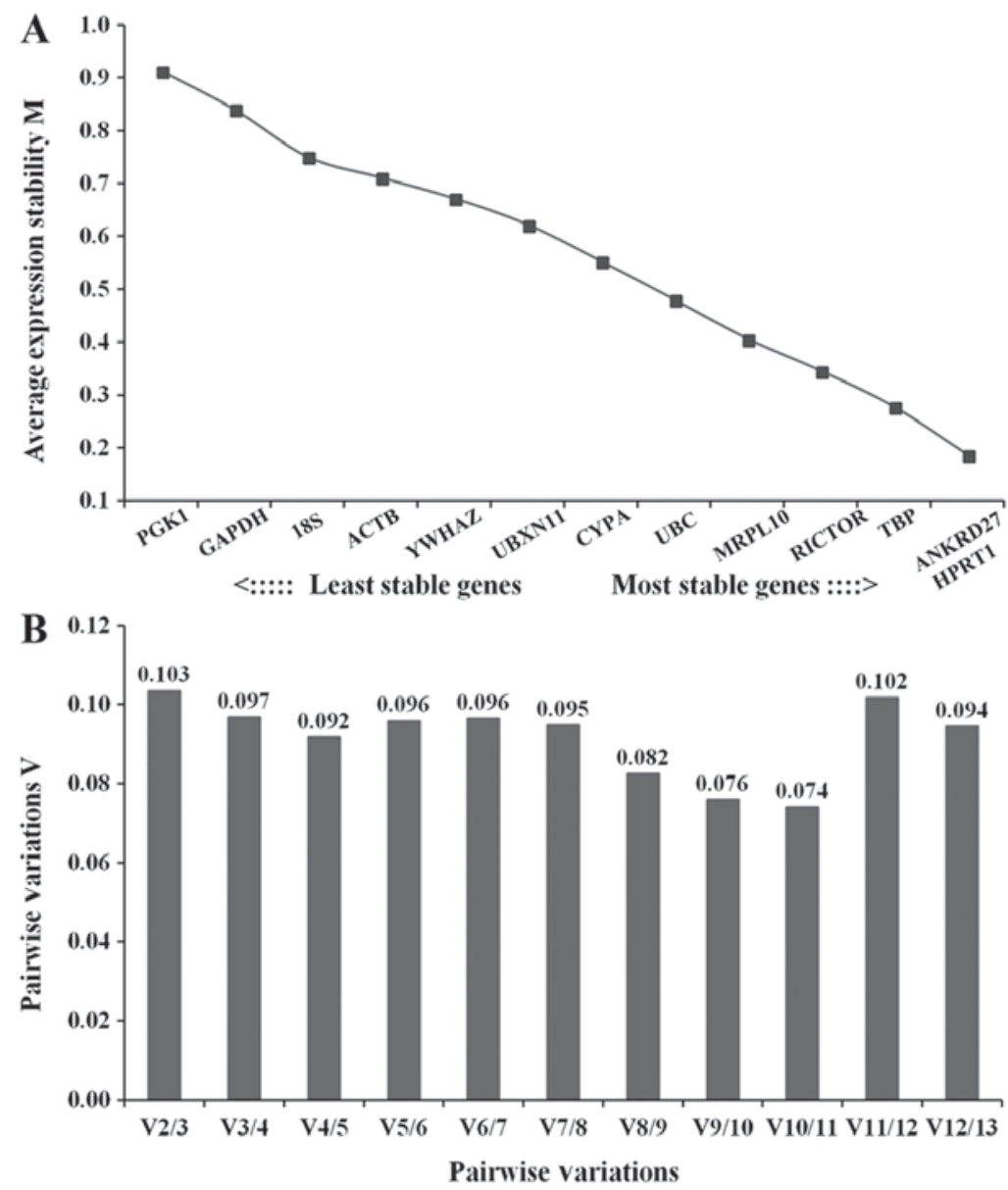

Figure 3. Analysis of candidate reference genes using geNorm. (A) Average expression stability of 18S, ACTB, ANKRD27, CYPA, GAPDH, HPRT1, MRPL10, PGK1, RICTOR, TBP, UBC, UBXN11 and YWHAZ. M values are inversely proportional to the stability of the expression levels of the corresponding genes. (B) Normalization factor (pairwise variation value, $\mathrm{Vn} / \mathrm{n}+1$ ) was calculated to assess the optimal numbers of reference genes to use for gene expression normalization. ANKRD27, ankyrin repeat domain 27; CYPA, cyclophilin A; HPRT1, hypoxanthine phosphoribosyltransferase 1; MRPL10, mitochondrial ribosomal protein L10; PGK1, phosphoglycerate kinase 1; RICTOR, RPTOR independent companion of mammalian target of rapamycin complex 2; TBP, TATA-box binding protein; UBC, ubiquitin C; UBXN11, UBX domain protein 11; YWHAZ, tyrosine 3-monooxygenase/tryptophan 5-monooxygenase activation protein $\zeta$; ACTB, actin $\beta$.

Results from geNorm analysis suggested that two reference genes were sufficient to normalize gene expression levels. In addition to HPRT1, ANKRD27 and TBP were identified to exhibit stable expression levels. Therefore, the geometric mean of ANKRD27 and TBP was used as an internal control (Fig. 5C and F). Using ANKRD27+TBP or HPRT1 as the internal controls did not affect the detected expression level of FBXO32, which exhibited similar fold changes at all time points following peripheral nerve injury (Fig. 5C).

Additionally, TRIM63 exhibited similar expression patterns at various time points following sciatic nerve injury, regardless of the reference gene selected (Fig. 5D-F). However, similarly to FBXO32, the calculated relative fold changes of TRIM63 varied depending on the reference gene selected. The expression level of TRIM63 at 4 days following nerve injury increased by $\sim 5$ fold when compared with normal control, using HPRT1 or ANKRD27+TBP as the internal control (Fig. 5D and F). By contrast, using PGK1 as the internal control, the detected relative expression level of TRIM63 at 4 days following nerve injury increased by $\sim 30$ folds when compared with the normal control (Fig. 5E).

\section{Discussion}

The denervation and reinnervation of target skeletal muscles is a biological process that occurs following peripheral nerve injury (10). Muscle denervation leads to muscle atrophy that is characterized by reduced muscle mass, altered muscle morphology and impaired muscle functions including decreased force production and power output (27-29). Clinical trials have demonstrated that reinnervation may reduce muscle atrophy and promote the recovery of muscle function. By contrast, prolonged muscle denervation may cause irreversible damage $(30,31)$.

Incomplete and/or delayed peripheral nerve reinnervation and consequent impaired muscle function remains a major health problem. Therefore, multiple previous studies investigated the alterations in gene expression during the process of muscle denervation and reinnervation following peripheral nerve injury, with the aim to identify novel therapeutic targets to treat patients with injury-induced muscle atrophy (32-35). Microarray analysis, a high-throughput method that allows parallel analysis of multiple samples, was used to identify gene expression profiles during muscle 


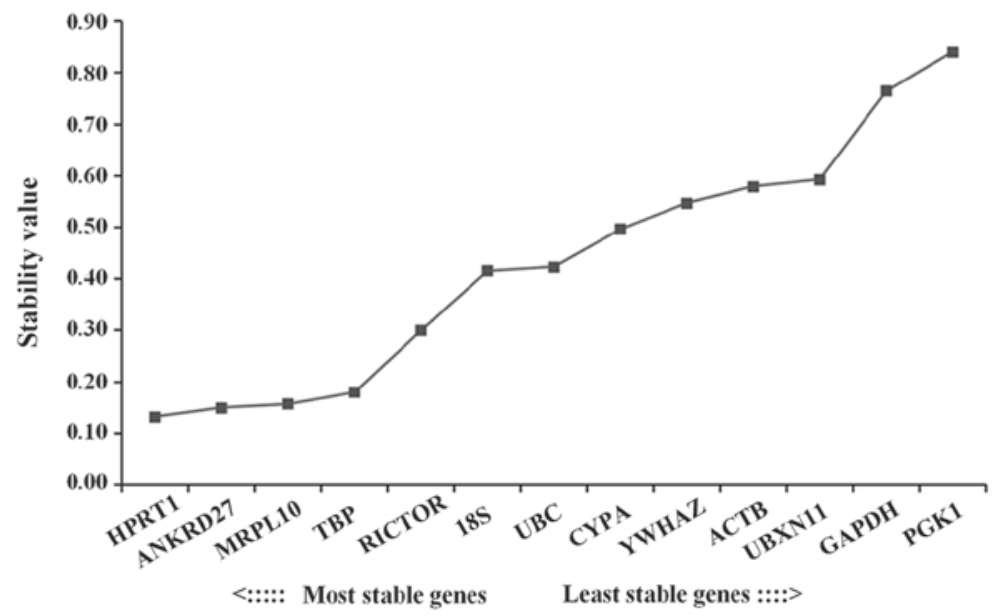

Figure 4. Analysis of candidate reference genes using NormFinder. Stability values of 18S, ACTB, ANKRD27, CYPA, GAPDH, HPRT1, MRPL10, PGK1, RICTOR, TBP, UBC, UBXN11 and YWHAZ. Stability values are inversely proportional to the stability of the expression levels of the corresponding genes. 18S, 18S ribosomal RNA; ANKRD27, ankyrin repeat domain 27; CYPA, cyclophilin A; HPRT1, hypoxanthine phosphoribosyltransferase 1; MRPL10, mitochondrial ribosomal protein L10; PGK1, phosphoglycerate kinase 1; RICTOR, RPTOR independent companion of mammalian target of rapamycin complex 2; TBP, TATA-box binding protein; UBC, ubiquitin C; UBXN11, UBX domain protein 11; YWHAZ, tyrosine 3-monooxygenase/tryptophan 5-monooxygenase activation protein $\zeta$; ACTB, actin $\beta$.

A

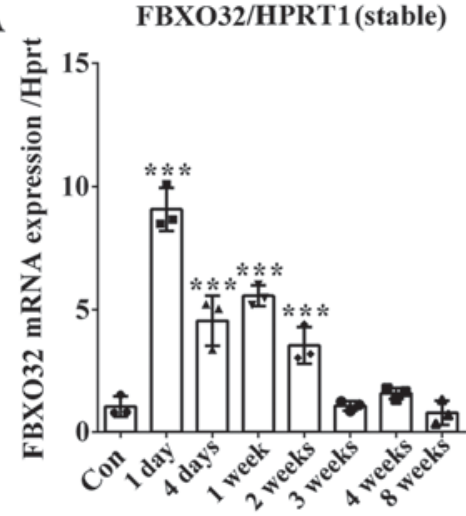

D

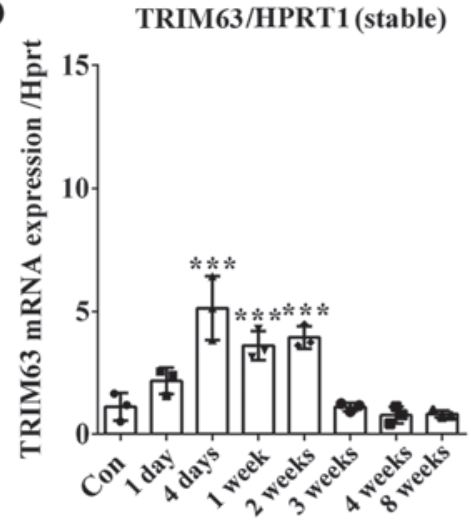

B

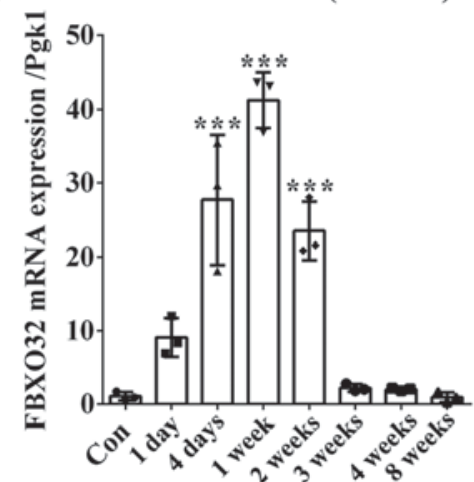

E

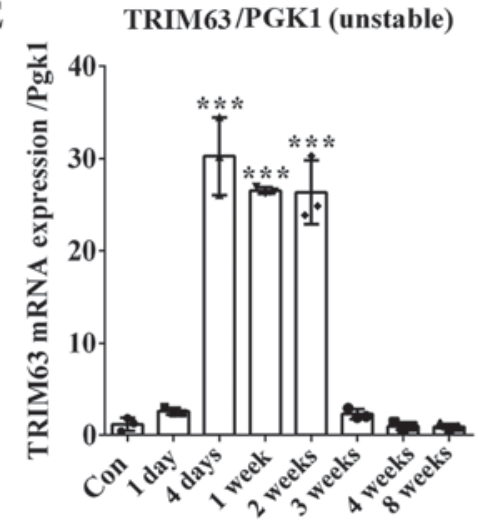

C FBXO32/(ANKRD27+TBP)

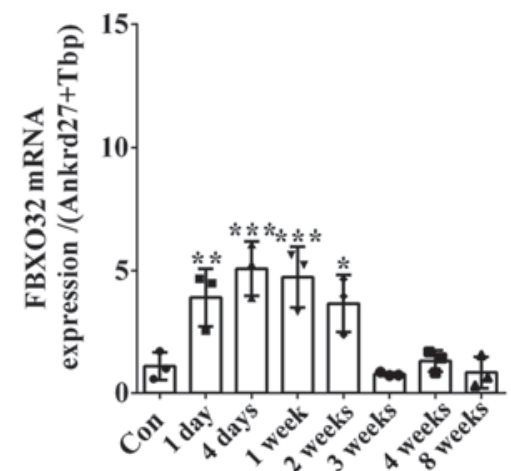

F TRIM63/(ANKRD27+TBP)

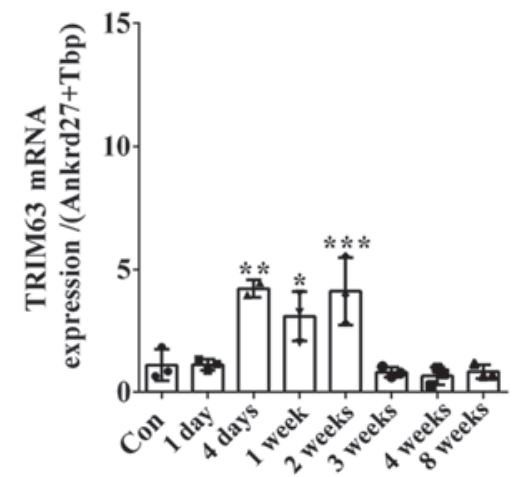

Figure 5. Determination of mRNA expression levels of FBXO32 and TRIM63 in gastrocnemius muscles using HPRT1, PGK1 or ANKRD27+TBP as reference genes. mRNA expression levels of FBXO32 were compared between normal control rats and rats subjected to sciatic nerve injury using (A) HPRT1, (B) PGK1 or (C) ANKRD27+TBP as the reference gene ( $\mathrm{n}=3$ in each group). mRNA expression levels of TRIM63 were compared between normal control rats and rats subjected to sciatic nerve injury using (D) HPRT1, (E) PGK1 or (F) ANKRD27+TBP as the reference gene (n=3 in each group). ${ }^{*}<0.05,{ }^{* *} \mathrm{P}<0.01$ and ${ }^{* * *} \mathrm{P}<0.001$ vs. Con. HPRT1, hypoxanthine phosphoribosyltransferase 1; PGK1, phosphoglycerate kinase 1; FBXO32, F-box protein 32; TRIM63, tripartite motif containing 63; ANKRD27, ankyrin repeat domain 27; TBP, TATA-box binding protein; Con, normal control rats.

denervation and reinnervation $(32,36)$. The expression levels of microRNAs and their target genes were also examined in the context of muscle reinnervation (37-39). The accuracy of the results derived from high-throughput approaches is frequently confirmed by RT-qPCR analysis. Therefore, the selection of suitable internal controls is required to decrease 
the inaccuracy derived by alterations in the expression levels of the reference gene and to increase the reliability of the RT-qPCR results.

In the present study, the morphological and physiological parameters in the gastrocnemius muscles were investigated in normal control rats and in rats following sciatic nerve crush injury, and structural and functional alterations were observed in the gastrocnemius muscles following nerve injury. Therefore, a great number of genes, including housekeeping genes, may be differentially expressed in gastrocnemius muscles during these biological processes. To examine the possibility that the expression levels of housekeeping genes may be affected, primer pairs with high efficiency were used and the raw $\mathrm{Cq}$ values of 13 housekeeping genes were measured in the control and injured rats. A subset of housekeeping genes, including 18S, ACTB, CYPA, GAPDH, PGK1, UBC, UBXN11 and YWHAZ, exhibited variable $\mathrm{Cq}$ values. The present results suggested that certain housekeeping genes may not exhibit stable expression levels in rat gastrocnemius muscles following sciatic nerve injury, further confirming the importance of the identification of a suitable reference gene to study this process.

By using geNorm and NormFinder analyses, the stabilities of these housekeeping genes were assessed. By using geNorm analysis, the housekeeping genes with the most stable expression levels were selected based on their expression level ratios $(2,6)$, and ANKRD27 and HPRT1 were identified to exhibit stable expression levels. By using NormFinder analysis, the gene expression variation and the similarities among expression profiles of the housekeeping genes were calculated (3), and the expression level of HPRT1 was identified as the most stable among the reference genes examined. geNorm and NormFinder results suggested that HPRT1 was the most suitable internal reference gene, whereas PGK1 was not a reliable internal control. RT-qPCR experiments were performed to analyze the expression profiles of FBXO32 and TRIM63 using various reference genes, in order to investigate whether the selection of a certain housekeeping gene may affect the quantification results. RT-qPCR results demonstrated that the expression patterns and fold changes of the examined target genes varied using the stable housekeeping gene HPRT1 or the unstable housekeeping gene PGK1 as the reference gene. Furthermore, the standard deviation values of target genes increased with PGK1 as the internal control. The present results suggested that the selection of the reference gene may influence experimental reproducibility. However, the genomic locus of HPRT1 presents pseudogenes (40), and the quantification of HPRT1 may be affected by genomic DNA contamination, despite treatment with DNase. Therefore, additional housekeeping genes with stable expression levels, including ANKRD27 and TBP, may be used. In the present study, the geometric mean of ANKRD27 and TBP was used to normalize the expression levels of FBXO32 and TRIM63. Quantitative results suggested that FBXO32 and TRIM63 exhibited similar temporal dynamics using HPRT1 or ANKRD27+TBP as the internal control, suggesting that ANKRD27+TBP may additionally be used as a suitable control.

Selection of suitable internal controls was previously identified to be involved in the accuracy of gene quantification. The selection of the most suitable reference gene was identified to be cell- and tissue-specific $(2,22,41,42)$. Our previous study suggested that distinct reference genes may be selected in sciatic nerve samples and DRG samples following rat sciatic nerve crush injury (13). In the present study, by using geNorm and NormFinder algorithms, the stabilities of the gene expression of 13 housekeeping genes were investigated, and HPRT1 was identified as the most stable housekeeping gene in gastrocnemius muscles following rat sciatic nerve crush injury. The present results suggested that the selection of an appropriate internal control is required for reliable gene normalization. Furthermore, the results of the present study may provide insights that may be beneficial for future studies aiming to analyze gene expression in innervated muscles following peripheral nerve injury.

\section{Acknowledgements}

The authors would like to thank Dr Sheng Yi at Nantong University (Nantong, China) for her assistance in manuscript preparation. The authors would also like to thank Professor Jie Liu at Nantong University (Nantong, China) for his help in language editing.

\section{Funding}

Thepresentstudy wassupported by TheNaturalScienceFoundation of Jiangsu Province (Jiangsu, China; grant no. BK20180951), The Natural Science Foundation of the Jiangsu Higher Education Institutions of China (Jiangsu, China; grant no. 18KJB180022), the Natural Science Research Project Funded by The Nantong University (Nantong, China; grant nos. 17ZY12 and 18B26) and the Priority Academic Program Development of Jiangsu Higher Education Institutions of China.

\section{Availability of data and materials}

The datasets used and/or analyzed during the present study are available from the corresponding author on reasonable request.

\section{Authors' contributions}

$\mathrm{XW}, \mathrm{HZ}$ and $\mathrm{YW}$ conceived and designed the present study. $\mathrm{XW}, \mathrm{HZ}, \mathrm{JN}$ and YW performed the experiments. JN, JP and $\mathrm{HH}$ analyzed the data. YW provided the reagents, materials, and analysis tools and interpreted the data. XW and YW wrote the manuscript. All authors read and approved the final manuscript.

\section{Ethics approval and consent to participate}

The animal procedures were ethically approved by The Administration Committee of Experimental Animals of Jiangsu Province (Jiangsu, China).

\section{Patient consent for publication}

Not applicable.

\section{Competing interests}

The authors declare that they have no competing interests. 


\section{References}

1. Bustin SA: Quantification of mRNA using real-time reverse transcription PCR (RT-PCR): Trends and problems. J Mol Endocrinol 29: 23-39, 2002.

2. Seol D, Choe H, Zheng H, Jang K, Ramakrishnan PS, Lim TH and Martin JA: Selection of reference genes for normalization of quantitative real-time PCR in organ culture of the rat and rabbit intervertebral disc. BMC Res Notes 4: 162, 2011.

3. Andersen CL, Jensen JL and Orntoft TF: Normalization of real-time quantitative reverse transcription-PCR data: A model-based variance estimation approach to identify genes suited for normalization, applied to bladder and colon cancer data sets. Cancer Res 64: 5245-5250, 2004.

4. Eisenberg E and Levanon EY: Human housekeeping genes are compact. Trends Genet 19: 362-365, 2003.

5. Zhu J, He F, Hu S and Yu J: On the nature of human housekeeping genes. Trends Genet 24: 481-484, 2008.

6. Vandesompele J, De Preter K, Pattyn F, Poppe B, Van Roy N, De Paepe A and Speleman F: Accurate normalization of real-time quantitative RT-PCR data by geometric averaging of multiple internal control genes. Genome Biol 3: RESEARCH0034, 2002.

7. Thellin O, Zorzi W, Lakaye B, De Borman B, Coumans B Hennen G, Grisar T, Igout A and Heinen E: Housekeeping genes as internal standards: Use and limits. J Biotechnol 75: 291-295, 1999.

8. Schmittgen TD and Zakrajsek BA: Effect of experimental treatment on housekeeping gene expression: Validation by real-time, quantitative RT-PCR. J Biochem Biophys Methods 46: 69-81, 2000.

9. Li R, Liu Z, Pan Y, Chen L, Zhang Z and Lu L: Peripheral nerve injuries treatment: A systematic review. Cell Biochem Biophys 68: 449-54, 2014

10. Gu X, Ding F and Williams DF: Neural tissue engineering options for peripheral nerve regeneration. Biomaterials 35 6143-6156, 2014.

11. Yi S, Zhang H, Gong L, Wu J, Zha G, Zhou S, Gu X and Yu B Deep sequencing and bioinformatic analysis of lesioned sciatic nerves after crush injury. PLoS One 10: e0143491, 2015.

12. Gong L, Wu J, Zhou S, Wang Y, Qin J, Yu B, Gu X and Yao C: Global analysis of transcriptome in dorsal root ganglia following peripheral nerve injury in rats. Biochem Biophys Res Commun 478: 206-212, 2016

13. Wang Y, Shan Q, Meng Y, Pan J and Yi S: Mrpl10 and Tbp are suitable reference genes for peripheral nerve crush injury. Int J Mol Sci 18: E263, 2017.

14. Lien SC, Cederna PS and Kuzon WM Jr: Optimizing skeletal muscle reinnervation with nerve transfer. Hand Clin 24: 445-454 vii, 2008.

15. Irintchev A and Wernig A: Denervation and reinnervation of muscle: Physiological effects. Eur Arch Otorhinolaryngol: S28-S30, 1994

16. Martínez-Martos JM, Carrera-González MP, Sánchez-Agesta R, García MJ and Ramírez-Expósito MJ: Kidney aminopeptidase activities are related to renal damage in experimental breast cancer. J Clin Mol Med 1, 2018.

17. Livak KJ and Schmittgen TD: Analysis of relative gene expression data using real-time quantitative PCR and the 2(-Delta Delta C(T)) method. Methods 25: 402-428, 2001.

18. Bangaru ML, Park F, Hudmon A, McCallum BJ and Hogan HQ Quantification of gene expression after painful nerve injury: Validation of optimal reference genes. J Mol Neurosci 46: 497-504, 2012

19. Bonefeld BE, Elfving B and Wegener G: Reference genes for normalization: A study of rat brain tissue. Synapse 62: 302-309, 2008 .

20. Gambarotta G, Ronchi G, Friard O, Galletta P, Perroteau I and Geuna S: Identification and validation of suitable housekeeping genes for normalizing quantitative real-time PCR assays in injured peripheral nerves. PLoS One 9: e105601, 2014.

21. Peinnequin A, Mouret C, Birot O, Alonso A, Mathieu J, Clarençon D, Agay D, Chancerelle Y and Multon E: Rat pro-inflammatory cytokine and cytokine related mRNA quantification by real-time polymerase chain reaction using SYBR green. BMC Immunol 5: 3, 2004.

22. Martínez-Beamonte R, Navarro MA, Larraga A, Strunk M, Barranquero C, Acín S, Guzman MA, Iñigo P and Osada J: Selection of reference genes for gene expression studies in rats. J Biotechnol 151: 325-334, 2011.
23. Carvalho RF, Castan EP, Coelho CA, Lopes FS, Almeida FL, Michelin A, de Souza RW, Araújo JP Jr, Cicogna AC and Dal Pai-Silva M: Heart failure increases atrogin-1 and MuRF1 gene expression in skeletal muscle with fiber type-specific atrophy. J Mol Histol 41: 81-87, 2010.

24. Rho HW, Lee BC, Choi ES, Choi IJ, Lee YS and Goh SH: Identification of valid reference genes for gene expression studies of human stomach cancer by reverse transcription-qPCR. BMC Cancer 10: 240, 2010

25. Li P, Waters RE, Redfern SI, Zhang M, Mao L, Annex BH and Yan Z: Oxidative phenotype protects myofibers from pathological insults induced by chronic heart failure in mice. Am J Pathol 170: 599-608, 2007.

26. Latres E, Amini AR, Amini AA, Griffiths J, Martin FJ, Wei Y, Lin HC, Yancopoulos GD and Glass DJ: Insulin-like growth factor-1 (IGF-1) inversely regulates atrophy-induced genes via the phosphatidylinositol 3-kinase/Akt/mammalian target of rapamycin (PI3K/Akt/mTOR) pathway. J Biol Chem 280: 2737-2744, 2005

27. Yoshimura K, Asato H, Cederna PS, Urbanchek MG and Kuzon WM: The effect of reinnervation on force production and power output in skeletal muscle. J Surg Res 81: 201-208, 1999.

28. Kamath S, Venkatanarasimha N, Walsh MA and Hughes PM MRI appearance of muscle denervation. Skeletal Radiol 37: 397-404, 2008

29. Jaweed MM, Herbison GM and Ditunno JF: Denervation and reinnervation of fast and slow muscles. A histochemical study in rats. J Histochem Cytochem 23: 808-827, 1975.

30. Carlson BM: The Biology of long-term denervated skeletal muscle. Eur J Transl Myol 24: 3293, 2014.

31. Willand MP, Nguyen MA, Borschel GH and Gordon T: Electrical stimulation to promote peripheral nerve regeneration. Neurorehabil Neural Repair 30: 490-496, 2016.

32. Batt J, Bain J, Goncalves J, Michalski B, Plant P, Fahnestock M and Woodgett J: Differential gene expression profiling of short and long term denervated muscle. FASEB J 20: 115-117, 2006.

33. Nicolino S, Panetto A, Raimondo S, Gambarotta G, Guzzini M, Fornaro M, Battiston B, Tos P, Geuna S and Perroteau I: Denervation and reinnervation of adult skeletal muscle modulate mRNA expression of neuregulin-1 and ErbB receptors. Microsurgery 29: 464-472, 2009.

34. Sun H, Qiu J, Chen Y, Yu M, Ding F and Gu X: Proteomic and bioinformatic analysis of differentially expressed proteins in denervated skeletal muscle. Int J Mol Med 33: 1586-1596, 2014.

35. Weng J, Zhang P, Yin X and Jiang B: The Whole transcriptome involved in denervated muscle atrophy following peripheral nerve injury. Front Mol Neurosci 11: 69, 2018.

36. Raffaello A, Laveder P, Romualdi C, Bean C, Toniolo L, Germinario E, Megighian A, Danieli-Betto D, Reggiani C and Lanfranchi G: Denervation in murine fast-twitch muscle: Short-term physiological changes and temporal expression profiling. Physiol Genomics 25: 60-74, 2006.

37. Hsieh CH, Jeng SF, Wu CJ, Lu TH, Yang JC, Chen YC, Lin CJ and Rau CS: Altered expression of the microRNAS and their potential target genes in the soleus muscle after peripheral denervation and reinnervation in rats. J Trauma 70: 472-480, 2011.

38. Jeng SF, Rau CS, Liliang PC, Wu CJ, Lu TH, Chen YC, Lin CJ and $\mathrm{Hsieh} \mathrm{CH}$ : Profiling muscle-specific microRNA expression after peripheral denervation and reinnervation in a rat model. J Neurotrauma 26: 2345-2353, 2009.

39. Li G, Li QS, Li WB, Wei J, Chang WK, Chen Z, Qiao HY, Jia YW, Tian JH and Liang BS: MIRNA targeted signaling pathway in the early stage of denervated fast and slow muscle atrophy. Neural Regen Res 11: 1293-1303, 2016.

40. Sellner LN and Turbett GR: The presence of a pseudogene may affect the use of HPRT as an endogenous mRNA control in RT-PCR. Mol Cell Probes 10: 481-483, 1996.

41. Nelissen K, Smeets K, Mulder M, Hendriks JJ and Ameloot M: Selection of reference genes for gene expression studies in rat oligodendrocytes using quantitative real time PCR. J Neurosci Methods 187: 78-83, 2010.

42. Solano ME, Thiele K, Kowal MK and Arck PC: Identification of suitable reference genes in the mouse placenta. Placenta 39: 7-15, 2016.

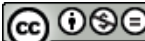

This work is licensed under a Creative Commons Attribution-NonCommercial-NoDerivatives 4.0 International (CC BY-NC-ND 4.0) License. 